\title{
Pelvic Irradiation for Stage II Ovarian Carcinoma
}

\author{
Keith Y. Terada, M.D., George W. Morley, M.D., \\ AND JAmes A. Roberts, M.D. \\ Department of Obstetrics and Gynecology, University of Michigan School of Medicine, \\ Ann Arbor, Michigan 48105
}

Received January 26, 1987

\begin{abstract}
Over a 20-year period, 34 patients with FIGO stage II ovarian carcinoma were treated with postoperative pelvic irradiation at the University of Michigan. Complications of radiation treatment were minimal. The overall actuarial disease-free 5-year survival was 53\%. This was not significantly different for substages IIA, IIB, or IIC. Patients with well-differentiated tumors had a significantly better survival than patients with moderate or poorly differentiated tumors $(P<0.05)$. The implications for managing stage II ovarian carcinoma are discussed. (c) 1988 Academic Press, Inc.
\end{abstract}

\section{INTRODUCTION}

Despite recent developments in the management of ovarian cancer, overall survival remains poor. Part of the reason for the low cure rate is that the majority of patients present with advanced stage disease. However, the recurrence risk even for patients with localized disease remains high. FIGO (International Federation of Gynecology and Obstetrics) stage II ovarian cancer is defined as growth involving one or both ovaries with pelvic extension. When managed by surgery alone the recurrence risk for stage II ovarian cancer is over $80 \%$ [1-3]. While it is clear that an initial optimal cytoreduction is important as primary treatment, ideal postoperative adjuvant therapy remains controversial $[4,5]$. This study was undertaken to review the 20-year experience at the University of Michigan Hospital in using postoperative pelvic irradiation in FIGO stage II ovarian carcinoma.

\section{MATERIALS AND METHODS}

Between January 1, 1961, and January 1, 1981, there were 38 patients treated for stage II carcinoma of the ovary at the University of Michigan Hosptial (see Table 1). All patients underwent an initial laparotomy for removal of the tumor; staging was based on findings at the time of the initial surgery. Twenty-eight of these patients had their initial operation performed at the University of Michigan; 10 patients had their initial operation performed at outside institutions and were subsequently referred for further management. Histologic sections on all patients were reviewed by a pathologist at the University of Michigan and all patients were presented for discussion at the Gynecology Tumor Conference. Three 
TABLE 1

Stage II Carcinoma of the Ovary (FIGO, Revised 1987)

Stage II

Stage IIA

Stage IIB

Stage IIC

\author{
Growth involving one or both ovaries with pelvic extension \\ Extension and/or metastases to the uterus and/or tubes \\ Extension to other pelvic tissues \\ Tumor either stage IIA or IIB, but with tumor on the surface of one or both \\ ovaries; or with capsule(s) ruptured; or with ascites present containing \\ malignant cells; or with positive peritoncal washings
}

patients received postoperative chemotherapy without radiation. One patient refused any adjuvant treatment. The remaining 34 patients all received postoperative pelvic irradiation. A retrospective review of the clinical course of these 34 patients forms the basis for the present study.

External pelvic irradiation was administered by the University of Michigan Radiation Therapy Department by a cobalt-60 machine. Anterior and posterior parallel opposed fields of 15 by $16 \mathrm{~cm}$ were utilized; a total midplane dose of approximately 5000 rad was administered in 5 to 6 weeks. All patients completed treatment without interruption. One patient also received a preoperative radium implant because of a separate primary adenocarcinoma of the endometrium. Seven patients received Chlorambucil, 6 to $10 \mathrm{mg}$ per day for 12 to 24 months following radiation therapy.

The ages of the patients ranged from 28 to 74 years with a mean of 52.3 years. All patients underwent a laparotomy to resect the primary tumor. In 29 patients an optimal cytoreduction was achieved (largest residual tumor less than $1.5 \mathrm{~cm}$ in diameter). A hysterectomy was performed in 24 patients and an omentectomy was performed in 7 patients. All patients underwent careful evaluation of the abdomen by inspection and palpation; however, it was generally not the practice during this period to carry out a complete staging procedure with retroperitoneal lymph node biopsies, omentectomy, subdiaphragmatic washings, etc.

The predominant types of ovarian cancer were epithelial in nature (see Table 2). The minimum follow-up for all patients was 5 years. No patient was lost to follow-up; four patients died of intercurrent causes during the 5-year period. $\chi^{2}$ analysis was used as a test of statistical significance.

\section{RESULTS}

The actuarial disease-free 5-year survival for 34 patients with stage II carcinoma of the ovary undergoing postoperative pelvic irradiation was $53 \%$. The survival in patients dying of their disease ranged from 9 to 72 months with a median of 25 months. When evaluated by substage the results are noted in Table 3 . The disease-free 5 -year survivals were $60 \%$ for stage IIA, $52 \%$ for stage IIB, and $52 \%$ for stage IIC. There was no significant difference in survival by substage. Patients with well-differentiated tumors had a significantly better outcome than patients with moderate or poorly differentiated tumors. Disease-free survivals at 5 -years were 78 and $30 \%$, respectively $(P<0.05)$. 
TABLE 2

Histology of Stage II Ovarian Cancers Undergoing Postoperative Pelvic Irradiation

\begin{tabular}{lc}
\hline Histology & $\begin{array}{c}\text { Number } \\
\text { of } \\
\text { patients }\end{array}$ \\
\hline Serous & 19 \\
Endometrioid & 9 \\
Mucinous & 3 \\
Clear cell & 1 \\
Carcinosarcoma & 1 \\
Unclassified sex cord stromal & 1 \\
\multicolumn{1}{c}{$\quad$ Total } & 34 \\
\hline
\end{tabular}

Of the five patients who underwent suboptimal cytoreduction at the time of their initial surgery (residual tumor greater than $1.5 \mathrm{~cm}$ in diameter) only one patient $(20 \%)$ was alive and free of disease at 5 years. Five-year survival was $56 \%$ for optimally resected patients; this difference was not statistically significant. In seven patients omentectomy and cytologic washings were performed; two patients also underwent pelvic and periaortic lymph node biopsies. Five-year survival for this optimally staged group was $46 \%$.

The survival in seven patients who underwent additional primary treatment with Chlorambucil was not significantly different; there were three of seven patients $(43 \%)$ alive and free of disease at 5 years.

The complications of postoperative pelvic irradiation were primarily of a minor nature (see Table 4). Twelve patients $(32 \%)$ were treated symptomatically for complaints of nausea, diarrhea, bladder irritation, or skin erythema. One patient (3\%) developed radiation enteritis with weight loss and intermittent episodes of a low grade bowel obstruction which responded to conservative management. There were no fistula complications and no patients required laprotomy for bowel complications.

TABLE 3

Follow-up in Patients with Stage II Ovarian Cancer at 5 Years

\begin{tabular}{cccccr}
\hline FIGO substage & A/NED & A/WD & D/WD & D/ICD & Total \\
\hline IIA & 3 & 0 & 2 & 0 & 5 \\
IIB & 6 & 1 & 5 & 1 & 13 \\
IIC & 6 & 0 & 7 & 3 & 16 \\
Total & 15 & 1 & 14 & 4 & 34 \\
\hline
\end{tabular}

Note. A/NED, alive/no evidence of disease; A/WD, alive/with disease; D/WD, dead/this cancer present at death; D/ICD, dead/of intercurrent causes. 
TABLE 4

COMPLiCATIONS OF RADIOTHERAPY IN 34

Patients Undergoing Pelvic Irradiation

\begin{tabular}{lc}
\hline Complication(s) & $\begin{array}{c}\text { Number } \\
\text { of } \\
\text { patients }\end{array}$ \\
\hline Radiation enteritis & 1 \\
Nausea, diarrhea & 6 \\
Skin erythema & 4 \\
Bladder irritation & 1 \\
\multicolumn{1}{c}{ Total } & 12 \\
\hline
\end{tabular}

\section{DISCUSSION}

Ovarian carcinoma is defined as stage II when tumor has spread beyond the ovaries, but remains confined to the pelvis. Although the cancer remains localized, survival rates are still poor. When surgery alone is utilized, long-term survival rates of less than $20 \%$ are obtained [1-3]. In the present series, postoperative pelvic irradiation resulted in a disease-free 5-year survival of 53\%.

Pelvic irradiation, however, fails to achieve long-term survival in a significant proportion of patients. One must consider the possibility of subclinical metastasis to the upper abdomen at the time of diagnosis. It was generally not the practice during most of the period of this study to perform complete staging procedures. Piver et al. have indicated that over $20 \%$ of patients diagnosed as having stage I and II ovarian cancer may be understaged at the time of surgery [6]. This clearly impacts upon survival. When sites of recurrence are noted following pelvic radiotherapy, the majority occur in the abdomen as well as in the pelvis [7,8]. Therefore, with stage II ovarian cancer, a careful surgical evaluation of the omentum, retroperitoneal nodes, bowel mesentery, and subdiaphragmatic area is crucial.

Dembo et al. [9] compared the results of using pelvic irradiation, pelvic plus whole abdomen irradiation, and pelvic irradiation plus Chlorambucil. For the combined group of Stage IB and II ovarian carcinoma the relapse-free survival at 5 years was $82 \%$ for patients receiving abdominopelvic irradiation, $52 \%$ for patients receiving pelvic irradiation plus Cholrambucil, and $50 \%$ for patients receiving pelvic irradiation alone. Therefore, abdominopelvic irradiation improves survival over pelvic irradiation alone or pelvic irradiation combined with Chlorambucil. The complications of abdominopelvic irradiation, however, may be significant. Fuks [3] noted that $78 \%$ of patients subjected to abdominal irradiation developed acute morbidity manifested by diarrhea, nausea, vomiting, and weight loss; $14 \%$ developed intestinal obstruction requiring laparotomy.

As an alternative to abdominal irradiation, some authors have recommended the intraperitoneal instillation of radiocolloids [2,5,10]. Pezner et al. [5] reports utilizing $15.0 \mathrm{mCi}$ of $\mathrm{P}^{32}$ combined with $4000 \mathrm{rad}$ pelvic irradiation. With this regimen there was a $67 \% 5$-year survival in patients with stages IIA and IIB 
with no gross residual tumor. However, with gross residual tumor left at the time of surgery, 5 -year survival was only $25 \%$ for stage IIB carcinoma. When external pelvic and intraperitoneal radiotherapy are combined, however, major bowel complications may occur in over $20 \%$ of patients $[11,12]$. Therefore, this particular combination may not provide a significant advantage in improving survival or reducing the risk of complications.

The role of chemotherapy in Stage II ovarian cancer is not clear. In the present study the addition of Chlorambucil did not improve results when compared to pelvic irradiation alone. Smith et al. [13] randomized patients with stages I, II, and III ovarian cancer between abdominopclvic irradiation versus Melphalan. Five-year survivals for stage II patients were $67 \%$ in the radiation group versus 73\% in the Melphalan group. Other studies comparing postoperative radiation with postoperative combination chemotherapy have found no significant difference in survival between the two groups $[14,15]$. Therefore postoperative adjuvant chemotherapy may be an effective alternative to radiotherapy in stage II ovarian carcinoma.

In summary, pelvic irradiation may be utilized as adjuvant therapy in patients with stage II ovarian carcinoma. A significant porportion of patients, however, may relapse following treatment; histologic grade may be of some benefit in predicting which patients will remain free of disease [16]. Patients that are incompletely staged may have subclinical stage III disease at the time of diagnosis and may be at particularly high risk for recurrence. Consideration should be given to treating these patients with chemotherapy or abdominopelvic radiotherapy. The initial surgical approach, therefore, should include an optimal resection of tumor in conjunction with a thorough exploration of the abdomen with multiple biopsies. The form of adjuvant treatment yielding optimal results remains undefined. An overall 5-year survival of 53\% indicates a limited efficacy when pelvic irradiation is used as the primary mode of adjuvant therapy following surgery.

\section{REFERENCES}

1. VanOrden, D., McAllister, W., Zerne, S., and Morris, J. Ovarian carcinoma, Amer. J. Obstet. Gynecol. 94, 195 (1966).

2. Clark, D. G. C., Hilaris, B., Roussis, C., and Brunschwig, A. The role of radiation therapy (including isotopes) in the treatment of cancer of the ovary (results of 614 patients treated at Memorial Hospital, New York, N.Y.), Prog. Clin. Cancer 5, 227 (1973).

3. Fuks, Z. The role of radiation therapy in the management of ovarian carcinoma, Israel J. Med. Sci. 13, 815 (1977).

4. Delclos, L., \& Quinlan, E. J. Malignant tumors of the ovary managed with postoperative megavoltage irradiation, Radiology 93, 659 (1969).

5. Pezner, R. D., Stevens, F. R., Tong, D., and Allen, C. V. Limited epithelial carcinoma of the ovary treated with curative intent by the intraperitoneal installation of radiocolloids, Cancer 42, 2563 (1978).

6. Piver, M. S., Barlow, J. J., and Lele, S. B. Incidence of subclinical metastasis in stage I and II ovarian cancer, Obstet. Gynecol. 52, 100 (1978).

7. Wharton, J., Smith, J., Delclos, L., and Fletcher, G. Radiation therapy for ovarian tumors, in (J. Sciarra, Ed.), Gynecology and Obstetrics Harper and Row, Philadelphia, Vol. 4, Chap. 37 (1985).

8. Wallner, P. E., Brady, I. W., Lewis, G. C., and Nuss, R. C. Postoperative pelvic irradiation of stage II ovarian carcinoma, Int. J. Radiat. Oncol. Biol. Phys. 2, 282 (1977). 
9. Dembo, F. J., Bush, R. S., Beale, F. A., Bean, H. A., Pringle, J. F., and Sturgeon, J. F. G. The Princess Margaret Hospital Study of ovarian cancer: Stages I, II, and asymptomatic III presentations, Cancer Treatment Rep. 63, 249 (1979).

10. Julian, C. G., Inalsingh, C. H., and Burnett, L. S. Radioactive phosphorus and external radiation as an adjuvant to surgery for ovarian carcinoma, Obstet. Gynecol. 52, 155 (1978).

11. Bakri, Y. N., Given, F. T., Peeples, W. J., and Frazier, A. B. Complications from intraperitoneal radioactive phosphorus in ovarian malignancies, Gynecol. Oncol. 21, 294 (1985).

12. Klaasen, O., Starreveld, A., Shelly, L. T., Miller, A., Boyes, D., Gerulath, A., and Levitt, M. External beam pelvic radiotherapy plus intraperitoneal radioactive chromic phosphate in early stage ovarian cancer: A toxic combination, Radiat. Oncol, Biol. Phys. 11, 1801 (1985).

13. Smith, J., Rutledge, F., and Delclos, L. Results of chemotherapy as an adjuvant to surgery in patients with localized ovarian cancer, Semin. Oncol. 2, 277 (1975).

14. Gronroos, M., Nieminen, U., Kauppila, A., Kauppila, O., Saksela, E., and Vayrynen, M. A prospective randomized national trial for treatment of ovarian cancer: The role of chemotherapy and external irradiation, Eur. J. Obstet. Gynecol. Reprod. Biol. 17, 33 (1984).

15. Menczer, J., Ben-Baruch, G., Modan, M., Brenner, J., and Brenner, H. A comparison of postoperative radiotherapy with postoperative chemotherapy in stage II-IV ovarian cancer patients, Gynecol. Oncol. 17, 207 (1984).

16. Mauch, P. M., Ehrmann, R. L., Griffiths, C. T., Marck, A., Knapp, R. C., and Levene, M. B. Radiation therapy in stage II ovarian carcinoma: The influence of histologic grade, Cancer 45, 1344 (1980). 